\title{
Collisional Effects on HFS Transitions of Antiprotonic Helium
}

\author{
G.Ya.Korenman*, N.P.Yudin, and S.N.Yudin \\ Institute of Nuclear Physics, Moscow State University, Moscow 119992, Russia
}

\begin{abstract}
Collisions of metastable antiprotonic helium with medium atoms induce transitions between hyperfine structure sublevels as well as shift and broadening of the microwave M1 spectral lines. We consider these effects in the framework of a simple model with scalar and tensor interactions between $\left(\bar{p} \mathrm{He}^{+}\right)_{n L}$ and He atoms. $S$ matrix is obtained by solving coupled-channels equations involving 4 HFS sublevels $(F=L \pm 1 / 2, J=F \pm 1 / 2)$ of the $n L$ level and relative angular momenta up to $l=5$ at the kinetic energy $E \lesssim 10 \mathrm{~K}$. The calculated spin-flip cross sections are less than elastic ones by 4 orders of value. At the density $N=3 \times 10^{20} \mathrm{~cm}^{-3}$ and $T=6$ $\mathrm{K}$ we obtain the relaxation times $\tau\left(F J \rightarrow F^{\prime} J^{\prime}\right) \gtrsim 160 \mathrm{~ns}$, the frequency shifts of M1 spectral lines $\Delta \nu \lesssim 66 \mathrm{KHz}$ for the favored transitions $(\Delta F= \pm 1, \Delta J= \pm 1)$ and frequency broadening of the M1 spectral lines $\gamma / 2 \lesssim 5.8 \mathrm{MHz}$. The results are compatible with the recent experimental data obtained by a laser-microwave-laser resonance method.
\end{abstract}

Key words: antiproton, antiprotonic helium, hyperfine structure, collisional relaxation, collisional shift and broadening

PACS: 36.10.-k, 34.60.+z, 32.70.Jz

The discovery of antiproton longevity in helium and investigations of antiprotonic helium $\left(\bar{p} \mathrm{He}^{+}\right)$metastable states by the method of laser resonance spectroscopy have opened a new layer of very interesting physics (see [1] and the references therein). One of the actual problems in the topics is an influence of ambient atoms on the antiprotonic states. In particular, the density shifts and broadenings of E1 spectral lines $\left(n L \rightarrow n^{\prime} L^{\prime}=L \pm 1\right)$ were observed for laserinduced transitions. The model theoretical analysis [2] shows that qualitative

\footnotetext{
* Corresponding author

Email addresses: korenman@anna19.npi.msu.su (G.Ya.Korenman), yudin@helene.sinp.msu.ru (N.P.Yudin), yudins@anna19.npi.msu.su (S.N.Yudin).
} 
peculiarities of the data are related to quantum effects at very low temperature and peculiar features of $\left(\bar{p} \mathrm{He}^{+}\right)-\mathrm{He}$ interaction. More sophisticated calculations with $a b$ initio potential surface [3] give a quantitative agreement with the experimental data for a lot of E1 transitions.

Recently, the first data on hyperfine structure of the $(n, L)=(37,35)$ state of $\left(\bar{p} \mathrm{He}^{+}\right)$were obtained by a laser-microwave-laser resonance method [4]. The central frequencies of microwave M1-transitions, $\nu_{H F}^{+}(F=L-1 / 2, J=L \rightarrow$ $L+1 / 2, L+1)=12.89596(34) \mathrm{GHz}$ and $\nu_{H F}^{-}(L-1 / 2, L-1 \rightarrow L+1 / 2, L)=$ $12.92467(29) \mathrm{GHz}$, are in excellent agreement $(\lesssim 30 \mathrm{ppm})$ with the recent calculations for the isolated $\left(\bar{p} H e^{+}\right)$system. The results suppose that density shifts of the M1 spectral lines at the experimental conditions are very small and do not exceed the experimental accuracy $(\sim 30 \mathrm{kHz})$, in contrast with the $E 1$ transitions, but the width of the lines $(\gamma \sim 5.3 \pm 0.7 \mathrm{MHz})$ leaves room for a collisional broadening. One more consequence of the data is that the mean time of collisional relaxation $\tau_{c}\left(F=L-1 / 2 \rightarrow F^{\prime}=L+1 / 2\right) \gtrsim 160$ ns, the latter number being the observation time window. As far as we know there is no published theoretical papers on collisional effects on HFS states of the $\left(\bar{p} \mathrm{He}^{+}\right)$system. We consider the problem in the frame of a simple model of $\left(\bar{p} \mathrm{He}^{+}\right)-$He potential [2] extended to the tensor interaction.

Hyperfine splitting of the $n L$ levels arises from interactions between orbital and spin magnetic momenta [5]. A coupling of orbital angular momentum $\mathbf{L}$ with spin of electron $\mathbf{s}_{e}$ splits each $n L$ level into two sublevels $F=L \pm 1 / 2$ with a displacement $\nu\left(F_{-}, F_{+}\right) \sim 13 \mathrm{GHz}$, each one, due to a coupling with spin of antiproton $\mathbf{s}_{\bar{p}}$ being in turn split into two subsublevel $J=F \pm 1 / 2$ with $\nu\left(F J_{+}, F J_{-}\right) \sim 150 \mathrm{MHz}$. To a first approximation, total wave functions of the split states is constructed by means of vector coupling of the angular momenta, $|n L F J M\rangle=\left(\left(\Psi_{n L}(\xi) \otimes \chi_{e}\right)_{F} \otimes \chi_{\bar{p}}\right)_{J M}$, where $\Psi_{n L \Lambda}(\xi)$ is a spatial wave function, $\chi_{e}$ and $\chi_{\bar{p}}$ are spin wave functions.

Interaction between antiprotonic helium in the state $|n L \Lambda\rangle$ and He atom with account for a tensor term is a matrix with respect to quantum numbers $\Lambda$ of projection of angular momentum $L$,

$$
\left\langle n L \Lambda|V(\mathbf{R}, \xi)| n L \Lambda^{\prime}\right\rangle=V_{0}(R) \delta_{\Lambda \Lambda^{\prime}}+V_{2}(R) \sum_{\nu}\left\langle L \Lambda^{\prime} 2 \nu \mid L \Lambda\right\rangle Y_{2 \nu}^{*}(\Omega) \sqrt{4 \pi / 5}
$$

where $\mathbf{R}=(R, \Omega)$ is a vector between centers of mass of the subsystems. Functions $V_{0}(R)$ and $V_{2}(R)$ depend on the quantum numbers $n, L$. For the scalar term we use [2] $V_{0}(R)=-C_{6} f(R)$ with $f(R)=\left(R^{2}-r_{c}^{2}\right) /\left(R^{2}+r_{0}^{2}\right)^{4}$ that has a repulsion at $R<r_{c}$, Van der Waals minimum at $R_{\min }^{2}=\left(r_{0}^{2}+4 r_{c}^{2}\right) / 3$ and the correct asymptotic $V_{0}(R) \rightarrow-C_{6} / R^{6}$ at $R \rightarrow \infty$. Radial dependence of the tensor term at large distance is similar, $V_{2}(R) \rightarrow-G_{6} / R^{6}$, whereas at small distance it has to be $V_{2}(R) \sim R^{2}$. To satisfy these limits we suppose $V_{2}(R)=-G_{6} f(R) \cdot\left[1-\exp \left(-\eta R^{2}\right]\right.$ with a large $\eta \sim 10$ a.u. For the calculations 
we use two sets of the parameters. The set $\mathrm{A}$ is based on the fitting of E1 shifts [2], $C_{6}=2.82, r_{c}=3.0, r_{0}=2.8$ (all values in atomic units). The second set $(\mathrm{B})$ is estimated using data of ab initio calculations of the potential energy surface [3,6]. A repulsion radius, position and depth of Van der Waals minimum of the potential $V_{0}(R)$ from Fig. 3 in [3] were used to obtain the values $C_{6}=3.35, r_{c}=4.75, r_{0}=0.707$ (a.u.) for our form of the potential. For the both sets we adopt $G_{6} / C_{6}=-0.37$ estimated by means of a single-particle model of $\left(\bar{p} \mathrm{He}^{+}\right)$with effective charges. A dependence of the parameters on $n, L$ is rather weak and does not matter for our aims. On other hand, two sets of the parameters differ markedly, that allows to reveal general properties of characteristics to be considered.

Second term in (1) is non-diagonal also with respect to HFS quantum numbers $F J$ and to a relative angular momentum $l$ of the interacting subsystems. Let $|n L F J, l: j m\rangle=\left(|n L F J\rangle \otimes Y_{l}(\Omega)\right)_{j m}$ be a state with total angular momentum $\mathbf{j}=\mathbf{J}+\mathbf{l}$ of the whole system. A matrix of the potentials (1) on this basis is reduced to the $3 j$ and $6 j$-symbols,

$$
\begin{array}{r}
\left\langle n L F J, l: j m|V(\mathbf{R}, \xi)| n L F^{\prime} J^{\prime}, l^{\prime}: j^{\prime} m^{\prime}\right\rangle=V_{0}(R) \cdot \delta_{j j^{\prime}} \delta_{m m^{\prime}} \delta_{F F^{\prime}} \delta_{J J^{\prime}} \delta_{l l^{\prime}} \\
+V_{2}(R) \cdot \delta_{j j^{\prime}} \delta_{m m^{\prime}} \hat{L} \hat{F} \hat{F}^{\prime} \hat{J} \hat{J}^{\prime} \hat{l} \hat{l}^{\prime}(-1)^{j+F+F^{\prime}+L+1} \\
\cdot\left(\begin{array}{lll}
l^{\prime} & 2 & l \\
0 & 0 & 0
\end{array}\right)\left\{\begin{array}{lll}
J & l & j \\
l^{\prime} & J^{\prime} & 2
\end{array}\right\}\left\{\begin{array}{ccc}
F & J & 1 / 2 \\
J^{\prime} & F^{\prime} & 2
\end{array}\right\}\left\{\begin{array}{ccc}
L & F & 1 / 2 \\
F^{\prime} & L^{\prime} & 2
\end{array}\right\},
\end{array}
$$

where $\hat{a} \equiv \sqrt{2 a+1}$. With these potentials, we solve coupled channels equations including 4 HFS sublevels at fixed $n L$ and relative angular momenta up to $l=5$ at the kinetic energy $E \lesssim 10 \mathrm{~K}$. Elastic and inelastic cross sections and rates of collisional transitions between HFS states are

$$
\begin{aligned}
\sigma\left(F J \rightarrow F^{\prime} J^{\prime}\right) & =\frac{\pi}{k^{2}} \sum_{j l l^{\prime}} \frac{2 j+1}{2 J+1}\left|\delta_{l l^{\prime}} \delta_{J J^{\prime}} \delta_{F F^{\prime}}-\left\langle F J l\left|S^{j}\right| F^{\prime} J^{\prime} l^{\prime}\right\rangle\right|^{2}, \\
\lambda\left(F J \rightarrow F^{\prime} J^{\prime}\right) & =N\left\langle v \sigma\left(F J \rightarrow F^{\prime} J^{\prime}\right)\right\rangle,
\end{aligned}
$$

where $\mathrm{N}$ is atomic density of the medium, and angular brackets stand for averaging over thermal velocity distribution. The results for the cross sections are shown on the Fig. 1. Non-diagonal elements of the S-matrix generated by the tensor term in (1) are small, therefore spin-flip cross sections are less than elastic one by 4 orders of value. Fig. 2 shows the calculated per-atom collisional transition rates averaged over the thermal velocity distribution.

Collisional shifts and broadenings of the microwave M1 transitions can be considered using a general theory of similar effects in atoms. For non-overlapping levels, Eq. (57.96) from [7] is relevant to our problem, and, with our notations 
of the quantum numbers, becomes

$$
\begin{aligned}
\gamma+\mathrm{i} \Delta & =N \pi \sum_{l l^{\prime} j_{1} j_{2}}\left(2 j_{1}+1\right)\left(2 j_{2}+1\right)(-1)^{l+l^{\prime}}\left\{\begin{array}{lll}
j_{1} & j_{2} & 1 \\
J_{2} & J_{1} & l
\end{array}\right\}\left\{\begin{array}{ccc}
j_{1} & j_{2} & 1 \\
J_{2} & J_{1} & l^{\prime}
\end{array}\right\} \\
& \cdot\left\langle v k^{-2}\left[\delta_{l l^{\prime}}-\left\langle n L F_{1} J_{1} l^{\prime}\left|S^{j_{1}}\right| n L F_{1} J_{1} l\right\rangle\left\langle n L F_{2} J_{2} l^{\prime}\left|S^{j_{2}}\right| n L F_{2} J_{2} l\right\rangle^{*}\right]\right\rangle,
\end{aligned}
$$

where S-matrix with the quantum numbers subscript 1 (2) corresponds to collisions before (after) M1 transition $\left(F_{1} J_{1} \rightarrow F_{2} J_{2}\right)$. Fig. 3 shows temperature dependence of shift and broadening for the transition $F=L-1 / 2, J=L \rightarrow$ $L+1 / 2, L+1$ calculated with the set $\mathrm{A}$ of the parameters.

At the experimental conditions $\left(T=6 \mathrm{~K}, N=3 \cdot 10^{20} \mathrm{~cm}^{-3}\right)$ we obtain from Fig. 2 a relaxation rate $\lambda\left(F_{-} \rightarrow F_{+}\right) \lesssim 6 \cdot 10^{6} \mathrm{~s}$, or $\tau \gtrsim 160 \mathrm{~ns}$. At the same conditions, shift and broadening of the M1 line from Fig. 3 are estimated as $\Delta \nu=65.8 \mathrm{KHz}$ and $\gamma / 2=5.8 \mathrm{MHz}$. In spite of rather simple used model, the results can be considered as compatible with upper limits of these value from the recent experimental data.

\section{References}

[1] T.Yamazaki, N.Morita, R.S.Hayano, E.Widmann and J.Eades, Physics Reports 366, 183 (2002).

[2] G.Ya.Korenman, Hyperfine Interactions, 119, 227 (1999).

[3] D.Bakalov, B.Jeziorski, T.Korona, K.Szalewich, Phys. Rev. Letters, 84, 2350 (2000).

[4] E.Widmann, R.S.Hayano, T.Ishikawa, J.Sakuraguchi, T.Tasaki, H.Yamaguchi, J.Eades, M.Hori, H.A.Torii, B.Juhasz, D.Horvath, and T.Yamazaki, Phys. Rev. Letters 89, 243402 (2002).

[5] D.Bakalov and V.Korobov, Phys. Rev. A 57, 1662 (1998); V.Korobov and D.Bakalov, J. Phys. B: At. Mol. Opt. Phys. 34, L519 (2001).

[6] S.Sauge and P.Valiron, Chemical Physics, 265, 47 (2001).

[7] G.Peach, Collisional broadening of spectral lines, in Atomic, Molecular and Optical Physics Handbook, ed. G.W.F.Drake, AIP Press, New York, 1996, Ch.57, p. 669. 


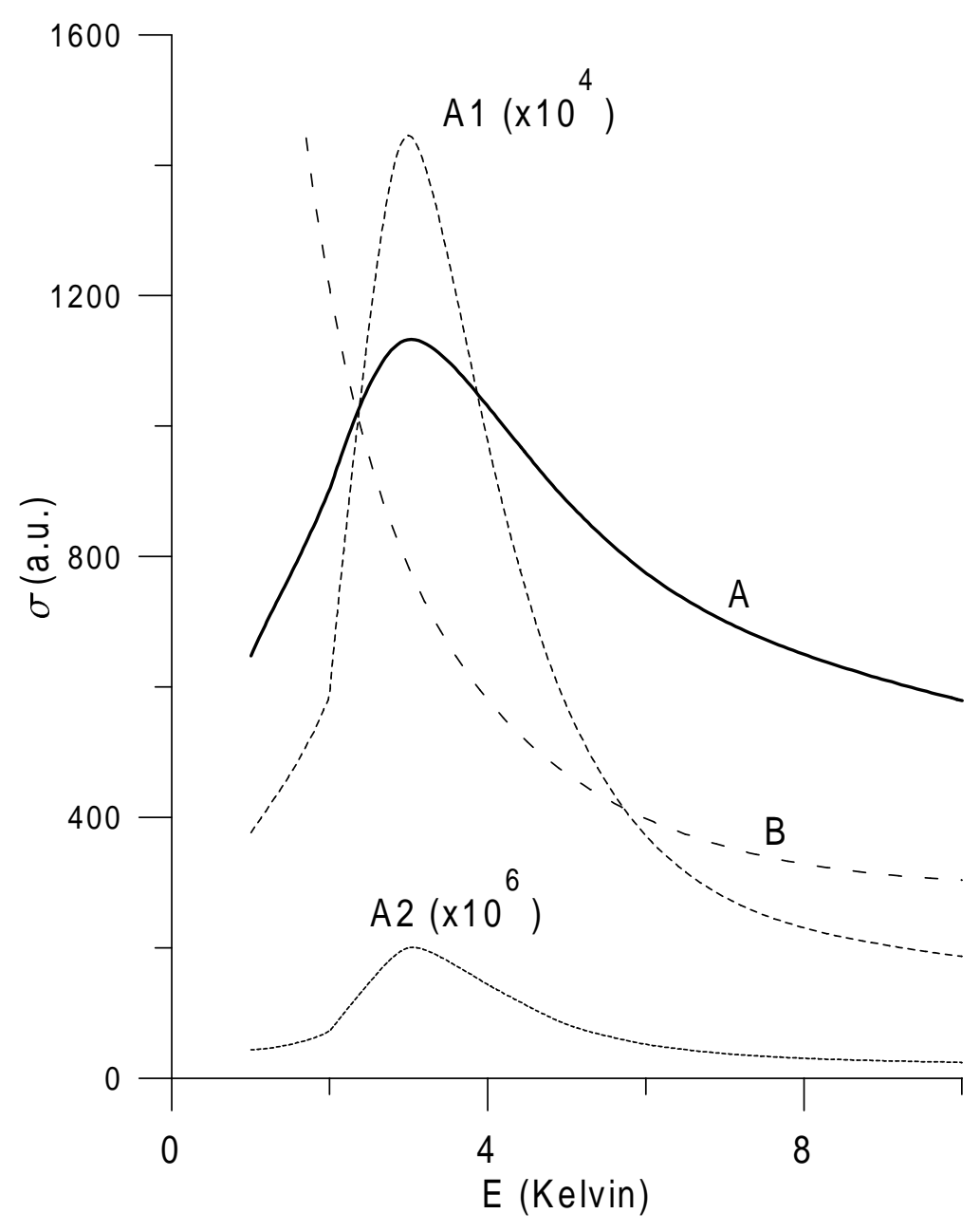

Fig. 1. Cross sections of $\left(\bar{p} \mathrm{He}^{+}\right)_{n L}-$ He collisions. Curves $A$ and $B$ are total cross sections averaged over HFS quantum numbers for the sets $\mathrm{A}$ and $\mathrm{B}$ of the parameters. Curves $A 1$ and $A 2$ are cross sections of transitions from $F, J=L-1 / 2, L$ to $L+1 / 2, L+1$ and $L+1 / 2, L$ multiplied by the factors $10^{4}$ and $10^{6}$, respectively. 


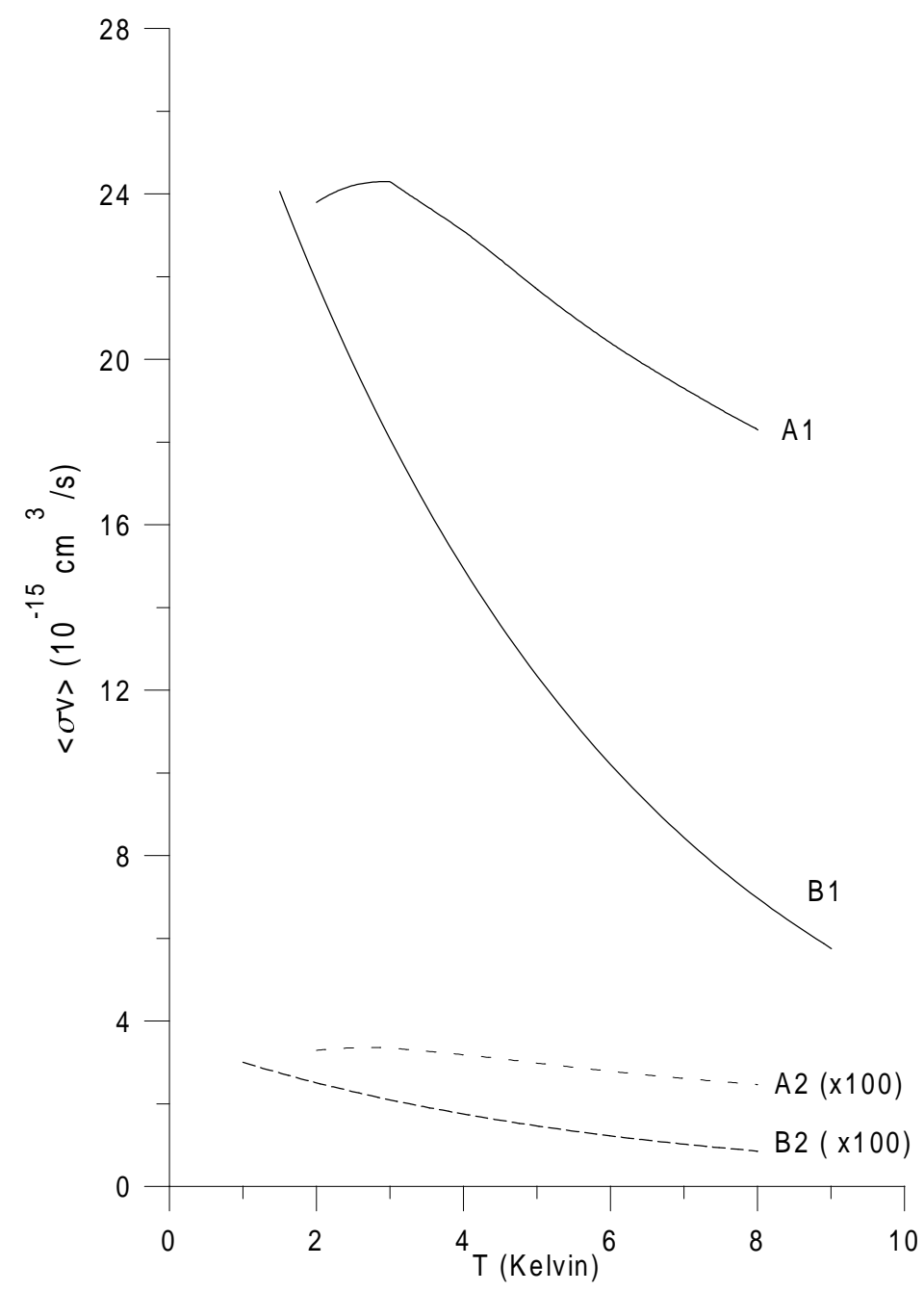

Fig. 2. Per-atom rate $\langle\sigma v\rangle$ of collisional transitions averaged over thermal motion depending on temperature. Curves $\mathrm{A} 1$ and $\mathrm{A} 2$ are for transition from $F=L-1 / 2, J=L$ to $L+1 / 2, L+1$ and $L+1 / 2, L$ with the parameters set $\mathrm{A}$, curves $\mathrm{B} 1$ and $\mathrm{B} 2$ are for the same transitions with the set $\mathrm{B}$, respectively. 


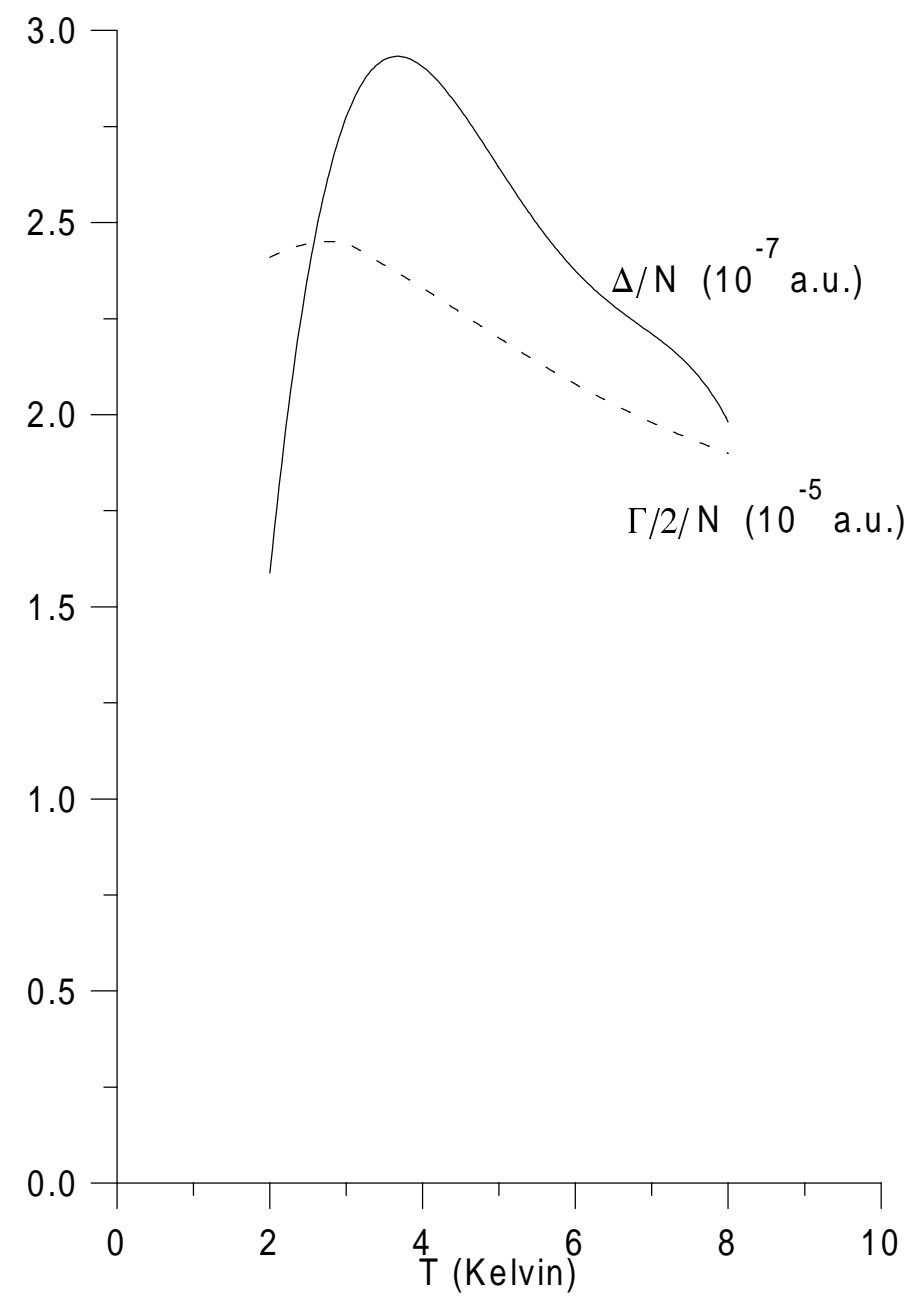

Fig. 3. Per-atom collisional shift and broadening of the M1 spectral line $\Delta(F=L-1 / 2, J=L \rightarrow L+1 / 2, L+1)$ depending on temperature. 